\title{
MODIFICATION SETS OF DENSITY ZERO ${ }^{1}$
}

\section{BY WALTER RUDIN}

Communicated by W. Wasow, December 18, 1967

Let $R, Z, T$ denote the real line, the integers, and the unit circle, respectively. A set $E \subset R$ will be called a modification set in $R$ if to every $f \in L^{1}(R)$ there corresponds a singular bounded Borel measure $\mu$ on $R$ whose Fourier transform $\hat{\mu}$ coincides with $\hat{f}$ in the complement of $E$. In other words, the Fourier transform of every absolutely continuous measure can be modified on $E$ alone so that the resulting function is the Fourier transform of a singular measure. Modification sets $E$ in $Z$ are defined similarly: to every $f \in L^{1}(T)$ there should correspond a bounded singular measure $\mu$ on $T$ whose Fourier coefficients satisfy $\hat{\mu}(n)=\hat{f}(n)$ for every integer $n$ which is not in $E$.

The existence of "small" modification sets in locally compact abelian groups has been established in [1]. However, when applied to $Z$ or $R$, the theorem of [1] can only yield modification sets of positive (though arbitrarily small) lower density. In the present note this result is improved to yield sets of density zero.

A set $E \subset R$ is said to have density zero if $(2 t)^{-1} m(E \cap[-t, t]) \rightarrow 0$ as $t \rightarrow \infty$, where $m$ denotes Lebesgue measure. If $E \subset Z$, the requirement is that the number of elements of $E$ in $[-N, N]$, divided by $2 N$, should tend to 0 as $N \rightarrow \infty$.

THEOREM 1. There are modification sets of density zero in $R$.

THEOREM 2. If $E$ is a modification set in $R$ then $E \cap Z$ is a modification set in $Z$.

THEOREM 3. There are modification sets of density zero in $Z$.

REMARK. Modification sets can of course not be too small. For instance, every modification set in $R$ has infinite measure (Plancherel); no lacunary set in $Z$ is a modification set; no set of positive integers is a modification set (F. and M. Riesz). On the other hand, largeness is not enough: Theorem 2 shows that the complement of $Z$ in $R$ is not a modification set.

Proof of Theorem 1. Choose integers $\lambda_{1}, \lambda_{2}, \lambda_{3}, \cdots$ so that $\lambda_{1}=10, \lambda_{k} \geqq 4 \lambda_{k-1}$. Let $A_{k}$ be the set of all numbers of the form

$$
\pm \lambda_{k}+\epsilon_{k-1} \lambda_{k-1}+\cdots+\epsilon_{1} \lambda_{1}
$$

${ }^{1}$ Research supported by National Science Foundation Grant GP-6764. 
where $\epsilon_{i}=1$ or 0 or -1 , let $B_{k}$ be the union of all intervals of length $2 k$ whose centers are in $A_{k}$, and put $E=B_{1} \cup B_{2} \cup B_{3} \cup \ldots$.

Given $t>10$, let $k=k(t)$ be the largest integer such that $\lambda_{k} \leqq 2 t$. Then $E \cap[-t, t] \subset B_{1} \cup \cdots \cup B_{k}$. Since $A_{i}$ has $2 \cdot 3^{i-1}$ points, $m\left(B_{i}\right)$ $\leqq 4 i \cdot 3^{i-1}$. Hence

$$
\frac{m(E \cap[-t, t])}{2 t} \leqq \frac{1}{2 t} \sum_{i=1}^{k} m\left(B_{i}\right)<\frac{2 k \cdot 3^{k}}{\lambda_{k}} \leqq \frac{4 k}{5} \cdot\left(\frac{3}{4}\right)^{b}
$$

which tends to 0 as $t$ (and hence $k$ ) tends to $\infty$. Thus $E$ has density zero.

For $k=1,2,3, \cdots$, let $\sigma_{k}$ be the measure on $T$ whose Fourier series is the formal expansion of the Riesz product

$$
d \sigma_{k}(x) \sim \prod_{j=k}^{\infty}\left(1+\cos \lambda_{j} x\right) .
$$

Then $\sigma_{k}$ is a bounded, positive, continuous, and singular measure on $T\left[2\right.$, p. 209] and $\hat{\sigma}_{k}(n)=0$ unless $n \in\{0\} \cup A_{k} \cup A_{k+1} \cup \ldots$.

Now choose $f \in L^{1}(R)$ so that $\hat{f}$ has compact support and two continuous derivatives. Then $x^{2} f(x)$ is bounded, so that $\sum|f(x-2 \pi j)|$, $j \in Z$, is a continuous periodic function. Fix $k$ so that $\hat{f}(t)=0$ whenever $|t|>k$. If we average the left side of (3) below over $-\pi \leqq s \leqq \pi$, and apply Fubini's theorem, we see that there exists an $s$ (fixed from now on) such that

$$
\int_{-\pi}^{\pi} \sum_{j=-\infty}^{\infty}|f(x-s-2 \pi j)| d \sigma_{k}(x) \leqq \int_{-\infty}^{\infty}|f(y)| d y=\|f\|_{1} .
$$

Define a measure $\mu$ on $R$ by requiring that

$$
\int_{-\infty}^{\infty} g d \mu=\int_{-\pi}^{\pi} \sum_{j=-\infty}^{\infty} g(x-s-2 \pi j) f(x-s-2 \pi j) d \sigma_{k}(x)
$$

for every bounded continuous $g$. Then $\mu$ is a singular measure on $R$ whose total variation satisfies $\|\mu\| \leqq\|f\|_{1}$, by (3). The Poisson summation formula now gives

$$
\begin{aligned}
& \int_{-\infty}^{\infty} e^{-i t x} d \mu(x)=\int_{-\pi}^{\pi} \sum_{j=-\infty}^{\infty} e^{-i t(x-s-2 \pi j)} f(x-s-2 \pi j) d \sigma_{k}(x) \\
&=\frac{1}{2 \pi} \int_{-\pi}^{\pi} \sum_{n=-\infty}^{\infty} \hat{f}(t-n) e^{-i n(x-s)} d \sigma_{k}(x)=\sum_{n=-\infty}^{\infty} \hat{\sigma}(n) \hat{f}(t-n) e^{i n s}
\end{aligned}
$$

which is the same as 


$$
\hat{p}(t)=\hat{f}(t)+\sum_{n \neq 0} \hat{\sigma}(n) \hat{f}(t-n) e^{i n s} \quad(t \in R) .
$$

In the last sum, $\hat{\sigma}(n)=0$ unless $n \in A_{k} \cup A_{k+1} \cup \cdots$, and $\hat{f}(t-n)=0$ if $|t-n| \geqq k$. Hence $\hat{a}(t)=\hat{f}(t)$ except possibly in $B_{k} \cup B_{k+1} \cup B_{k+2}$ $\cup \ldots$ which is a subset of $E$.

To conclude the proof, let $f$ be an arbitrary member of $L^{1}(R)$. Then $f=\sum f_{n}$ where $\sum\left\|f_{n}\right\|_{1}<\infty$ and each $\hat{f}_{n}$ has compact support and two continuous derivatives. The preceding step shows that there are singular measures $\mu_{n}$ with $\left\|\mu_{n}\right\| \leqq\left\|f_{n}\right\|_{1}$, such that $\hat{\mu}_{n}(t)=\hat{f}_{n}(t)$ outside $E$. The series $\sum \mu_{n}$ then converges in the total variation norm to a measure $\mu$ which is therefore also singular, and if $t$ is not in $E$ we have

$$
\hat{\mu}(t)=\sum \hat{\mu}_{n}(t)=\sum \hat{f}_{n}(t)=f(t) .
$$

Thus $E$ is a modification set in $R$.

Proof of Theorem 2. Let $E$ be a modification set in $R$. Choose $f \in L^{1}(T)$, regard $f$ as a member of $L^{1}(R)$ which vanishes outside $[-\pi, \pi)$, and let $\mu$ be a singular measure on $R$ such that $\hat{\mu}(t)=\hat{f}(t)$ outside $E$. For $V \subset[-\pi, \pi)$, define $\sigma(V)=\sum \mu(V-2 \pi j), j \in Z$. Then $\sigma$ is a singular measure on $T$, and $\hat{\sigma}(n)=\hat{\mu}(n)$ for every $n \in Z$. If $n \in Z$ and $\hat{f}(n) \neq \hat{\sigma}(n)$ it follows that $n \in E \cap Z$. So $E \cap Z$ is a modification set in $Z$.

Proof of Theorem 3. If $E$ is one of the sets constructed in the proof of Theorem 1 then $E \cap Z$ has density zero in $Z$. Hence Theorem 3 follows from Theorem 2 .

\section{REFERENCES}

1. Walter Rudin, Modifications of Fourier transforms, Proc. Amer. Math. Soc. (to appear).

2. Antoni Zygmund, Trigonometric series, 2nd ed., Vol. I, Cambridge Univ. Press, New York, 1959.

UNIVERSITY OF WISCONSIN 